\title{
Rupturas del tendón del supraespinoso: correlación entre RMN y hallazgos quirúrgicos
}

\author{
Supraspinatus tendon ruptures: correlation between MRI \\ and surgical findings
}

\author{
Salinas-Vela LE, * Aguirre-Rodríguez VH, ${ }^{\ddagger}$ Palmieri-Bouchan RB, ${ }^{*}$ \\ Encalada-Díaz MI, ${ }^{\ddagger}$ Mejía-Terrazas GE, ${ }^{\S}$ Valero-González FS
}

Clínica de Reconstrucción Articular Hombro y Codo. Hospital Ángeles Pedregal. CDMX.

RESUMEN. Introducción: En la ruptura del manguito de los rotadores, el tendón del supraespinoso ocupa el primer lugar en frecuencia. La resonancia magnética es el estudio de elección para el diagnóstico y planificación preoperatoria. El objetivo de este estudio fue evaluar la concordancia entre los hallazgos observados con la IRM y los hallazgos transoperatorios en pacientes con ruptura del tendón del supraespinoso. Material y métodos: Se realizó un análisis retrospectivo de Enero de 2014 a Enero de 2020. Se incluyeron pacientes mayores de 18 años, con IRM y reporte de ruptura del tendón del supraespinoso. Se realizó un análisis de $\chi^{2}$ para la sensibilidad, especificidad, valores predictivos y certeza diagnóstica utilizando los hallazgos quirúrgicos como referencia. Se utilizó el índice de Kappa para mostrar la concordancia entre IRM y hallazgos transoperatorios. Resultados: Un total de 79 pacientes se incluyeron en el estudio, 45 masculinos y 34 femeninos. La edad promedio fue de 52.14 años. La IRM diagnosticó correctamente 60.76\% de las rupturas del supraespinoso, mostró una sensibilidad de $74 \%$ y especificidad de $96 \%$ para rupturas completas. Para rupturas parciales mostró una sensibilidad de $96 \%$ y una especificidad de 33\%. El índice de Kappa mostró una concordancia de 0.90 para rupturas totales y de 0.53 para rupturas parciales. Conclusiones: La resonancia magnéti-
ABSTRACT. Introduction: In rotator cuff rupture, the supraspinatus tendon ranks first in frequency. MRI is the study of choice for preoperative diagnosis and planning. The objective of this study was to assess the concordance between findings observed with MRI and transoperative in patients with supraspinatus tendon rupture. Material and methods: A retrospective analysis was conducted from January 2014 to January 2020. Including patients over the age of 18 , with MRI and supraspinatus tendon rupture report. A $\chi^{2}$ analysis was performed for sensitivity, specificity, predictive values and diagnostic certainty using surgical findings as a reference. The kappa index was used to show the concordance between MRI and transoperative findings. Results: A total of 79 patients were included in the study, 45 male and 34 female. The average age was 52.14 years. MRI correctly diagnosed $60.76 \%$ of supraspinatus ruptures, showing $74 \%$ sensitivity and $96 \%$ specificity for complete ruptures. For partial ruptures I show a sensitivity of $96 \%$, a specificity of $33 \%$. The kappa index showed a match of 0.90 for total ruptures and 0.53 for partial. Conclusions: MRI demonstrated good sensitivity and specificity for diagnosing complete ruptures, with good match to surgical findings. MRI proved to be a non-

\section{Nivel de evidencia: IV, Estudio retrospectivo.}

* Alumno del Curso de Alta Especialidad en Cirugía de Reconstrucción Articular Hombro y Codo.

${ }^{\ddagger}$ Profesor Asociado del Curso de Alta Especialidad en Cirugía de Reconstrucción Articular Hombro y Codo.

§ Servicio de Anestesiología y Clínica del Dolor, Hospital Ángeles México.

" Profesor Titular del Curso de Alta Especialidad en Cirugía de Reconstrucción Articular Hombro y Codo.

Facultad Mexicana de Medicina de la Universidad La Salle-Hospital Ángeles Pedregal-GASS.

Correspondencia:

Dr. Fernando Sergio Valero González

Camino a Santa Teresa Núm. 1055-970, Col. Héroes de Padierna, C.P. 10700. Alcaldía Magdalena Contreras, CDMX.

E-mail: shoulder.elbow@gmail.com

Citar como: Salinas-Vela LE, Aguirre-Rodríguez VH, Palmieri-Bouchan RB, Encalada-Díaz MI, Mejía-Terrazas GE, Valero-González FS. Rupturas del tendón del supraespinoso: correlación entre RMN y hallazgos quirúrgicos. Acta Ortop Mex. 2020; 34(6): 399-402. https://dx.doi.org/10.35366/99138 
ca demostró una buena sensibilidad y especificidad para el diagnóstico de rupturas completas, con una buena concordancia con los hallazgos quirúrgicos. La IRM demostró ser un estudio poco específico para la identificación de rupturas parciales, lo cual genera que estas lesiones estén sobrediagnosticadas.

Palabras clave: Resonancia magnética, manguito de los rotadores, rupturas completas, rupturas parciales, supraespinoso.

\section{Introducción}

El hombro doloroso puede causar discapacidad hasta para realizar actividades de la vida diaria; se estima que tiene una prevalencia de $16-26 \%$ y es la tercera causa más común de consulta musculoesquelética. ${ }^{1}$ La causa más frecuente de hombro doloroso son las afecciones del manguito de los rotadores (MR), se puede presentar de manera aguda debido a traumatismo directo al hombro o bien puede ser crónica secundaria al proceso degenerativo de los tendones del MR, causando pérdida de la estructura y función de los mismos. La ruptura del manguito de los rotadores (RMR) afecta aproximadamente a $40 \%$ de la población adulta $>60$ años, ${ }^{2}$ causando un impacto económico de \$3,000 millones de dólares por año en costos de atención y pérdida de productividad en EUA. ${ }^{3}$ De los cuatro tendones que conforman el MR, el tendón del músculo supraespinoso es el más frecuentemente afectado.

Dentro de la ruta diagnóstica de los pacientes con ruptura del tendón del músculo supraespinoso, la realización de una imagen de resonancia magnética (IRM) es clave para decidir el plan de tratamiento. ${ }^{4}$ La IRM parece ser el estudio ideal para evaluar la patología del MR, específicamente la ruptura del tendón músculo supraespinoso, ya que ha demostrado tener una sensibilidad (90-95\%) y especificidad (85-90\%) alta, con una adecuada relación costo-efectividad, no emite radiación y no es invasivo. ${ }^{5,6,7}$ Provee información sobre la extensión, el tamaño, la retracción y la configuración de la ruptura del tendón e identifica si la ruptura es parcial o completa. ${ }^{4,8}$

A pesar de lo anterior, en ocasiones nos encontramos con rupturas que pasaron inadvertidas en la imagen o bien se sobrediagnostica la ruptura del tendón del músculo supraespinoso, ambas situaciones producen una alteración del plan quirúrgico. ${ }^{9}$

El objetivo del estudio fue evaluar la concordancia diagnóstica de la IRM con los hallazgos quirúrgicos en pacientes con rupturas del tendón del músculo supraespinoso.

\section{Material y métodos}

Se realizó un estudio retrospectivo donde se revisaron expedientes clínicos y reportes radiológicos de Enero de specific study for the identification of partial ruptures, which causes these lesions to be overdiagnosed.

Keywords: Magnetic resonance imaging, rotator cuff, complete ruptures, partial ruptures, supraspinatus.

2014 a Enero de 2020 de la Clínica de Reconstrucción Articular de Hombro y Codo de nuestro hospital.

Se incluyeron pacientes mayores de 18 años, de ambos géneros, que contaran con estudio de IRM, el cual tuviera una realización máxima de seis meses previos a la cirugía. Se tomaron en cuenta los diagnósticos de ruptura parcial o completa del tendón del músculo supraespinoso, se tomaron los diagnósticos emitidos por el radiólogo especialista y que subsecuentemente fueran tratados de manera quirúrgica. Todos los procedimientos quirúrgicos fueron realizados por el mismo equipo quirúrgico, los hallazgos transquirúrgicos se recabaron de la nota postoperatoria. Los hallazgos transoperatorios se correlacionaron con el diagnóstico establecido por el médico radiólogo.

Se excluyeron pacientes con cirugía de hombro realizada en otra institución, cirugía de revisión, diagnósticos de tendinitis calcificada del supraespinoso, artritis glenohumeral avanzada así como pacientes que reportaron en la imagen de resonancia magnética rupturas intrasustancia del supraespinoso.

Para definir quirúrgicamente las rupturas como completas o parciales se usó la clasificación de Ellman. ${ }^{10}$

La IRM utilizada cuenta con secuencias en los tres planos (coronal, sagital y axial), en T1, T2, saturación grasa y densidad protónica. Todos los hallazgos fueron reportados por un radiólogo especialista en patología musculoesquelética y fueron clasificados como rupturas parciales y rupturas completas del supraespinoso. El criterio para definir las rupturas completas del supraespinoso fueron áreas focales, bien definidas de incremento de señal en T1 y T2 que se extendieran desde la superficie bursal hasta la articular. Una ruptura parcial se define cuando no hay comunicación entre la articulación glenohumeral y el espacio subacromial.

Análisis estadístico: se realizó un análisis descriptivo de las variables demográficas. Se realizó un análisis con $\chi^{2}$ para evaluar la sensibilidad, especificidad, valores predictivos negativos y positivos y la certeza diagnóstica. Se consideró una $\mathrm{p}<0.05$ como estadísticamente significativa. La prueba de Kappa se utilizó para valorar la concordancia entre los hallazgos de la resonancia magnética y los hallazgos transoperatorios. Los valores de $\mathrm{k}$ fueron interpretados de la siguiente manera: 0.81-100 muy buena concordancia; 0.610.80 buena concordancia; $0.41-0.60$ concordancia mode- 
rada; 0.21-0.40 concordancia débil; y 0.00-0.20 mala concordancia. Se utilizó el Software SPSS ${ }^{\circledR}$ versión 16 (SPSS Inc., Chicago, IL) para todos los cálculos.

\section{Resultados}

En el estudio se evaluó un total de 732 expedientes, donde se incluyó un total de 79 pacientes que cumplían con los criterios de inclusión, de los cuales 45 fueron masculinos (56.96\%) y 34 femeninos (43.04\%) con una relación 1.3:1. La edad promedio fue de 52.14 años con un rango de 22-78 ( \pm 13.14$)$. Se operaron 48 hombros derechos y 31 hombros izquierdos. El lado dominante estuvo afectado en $63.29 \%$ de los casos.

De los 79 pacientes incluidos en el estudio, 60 de ellos (75.95\%) fueron diagnosticados con ruptura parcial del supraespinoso y 19 (24.05\%) con ruptura completa del supraespinoso por IRM. Los hallazgos transoperatorios reportaron 25 rupturas parciales (31.64\%), 23 rupturas completas $(29.11 \%)$ y 31 pacientes presentaron un tendón normal (39.24\%). En general la resonancia magnética sólo fue capaz de diagnosticar correctamente $60.76 \%$ (48/79) de las rupturas del supraespinoso.

Para rupturas parciales, la IRM diagnosticó correctamente 25 de 60 casos (41.67\%) y para rupturas completas diagnosticó 19 de 23 casos, la correlación entre IRM y hallazgos quirúrgicos se muestra en la Tabla 1. Al realizar la concordancia con el índice Kappa, se encontró que para las rupturas parciales fue de 0.53 , lo que es una concordancia moderada y para las rupturas completas la concordancia fue de 0.90 , la cual se considera muy buena.

\section{Discusión}

Dentro de la patología del hombro, la RMR ocupa un lugar importante por su frecuencia, controversia en su tratamiento y pronóstico, el tendón del supraespinoso es el que más frecuentemente se lesiona. ${ }^{11}$ De forma similar al estudio de Yamamoto y colaboradores en el cual se reportó que las rupturas del manguito rotador están asociadas con la edad avanzada, población masculina y en extremidad dominante, ${ }^{12}$ en nuestro estudio la ruptura de supraespinoso por resonancia fue más común en pacientes del género masculino en una relación 1.3:1. El hombro derecho así como el hombro dominante fueron afectados con mayor frecuencia. Encontramos que la mayoría de las rupturas de supraespinoso estaban presentes en pacientes mayores de 40 años. La edad avanzada es un factor de riesgo de degeneración tendinosa, la cual predispone a ruptura. ${ }^{13}$
En nuestro estudio las rupturas parciales se observaron en $31.64 \%$ pacientes y las rupturas totales en $29.11 \%$ pacientes, el restante $39.24 \%$ de los pacientes presentaron un tendón normal usando los hallazgos quirúrgicos como referencia. Se observó una alta sensibilidad y especificidad de la IRM para rupturas completas y una alta sensibilidad, pero una baja especificidad para rupturas parciales. Existen estudios en los que la sensibilidad y especificidad en las rupturas completas son equiparables con los resultados de nuestra investigación como el estudio de Jesus JO y colaboradores, el cual menciona que la resonancia magnética tiene buena sensibilidad y especificidad para la detección de rupturas completas. ${ }^{14}$ Tuite y su equipo evaluaron la sensibilidad y especificidad de la resonancia magnética para detectar rupturas de supraespinoso en secuencias de T2 y en supresión de grasa y demostraron una sensibilidad y especificidad de 90 y $91 \%$ respectivamente. ${ }^{15}$

Rafii y colaboradore ${ }^{16}$ demostraron una sensibilidad y especificidad altas, 89 y $85 \%$ respectivamente para rupturas parciales. En nuestro estudio se observó una alta sensibilidad, pero baja especificidad, diferente a lo evidenciado en el estudio de Reinus y su equipo, ${ }^{17}$ el cual demostró una baja sensibilidad (57\%) y en el estudio de Jesus JO y colaboradores, donde se observó una sensibilidad y especificidad de 64 y $92 \%$ respectivamente.

Nuestro estudio evidenció una certeza diagnóstica de $90 \%$ para rupturas totales muy similar al estudio de Sharma y colegas, el cual demostró una certeza diagnóstica de 91.1\% para rupturas totales. La IRM es eficaz para la detección de rupturas completas, por lo que el uso de manera preoperatoria puede ayudar a determinar el patrón de ruptura y planificar el mejor método de reparación, ${ }^{9,18}$ no así en las rupturas parciales donde la resonancia tuvo una certeza diagnóstica de $53 \%$.

La razón de la baja identificación de lesiones parciales por resonancia magnética aún no es clara, lo que refleja su incapacidad para distinguir áreas de mayor señal debido al proceso inflamatorio derivado de los desgarros parciales. ${ }^{19}$ Raffi y colaboradores señalaron una falta de intensidad de la señal en las secuencias en T2 al nivel de lesión en 50\% de las lesiones parciales. ${ }^{20}$ Algunos autores han observado que las lesiones de la superficie de la bursa eran superficiales y podían interpretarse como degeneración parcial del tendón o como lesiones degenerativas, mientras que las lesiones intrasustancia pueden aparecer como fisuras dentro de áreas degeneradas o infiltradas del tendón reemplazadas por tejido conectivo. ${ }^{21}$

\begin{tabular}{|c|c|c|c|c|c|c|c|c|c|}
\hline & Sensibilidad & Especificidad & $\begin{array}{c}\text { Falsos } \\
\text { positivos }\end{array}$ & $\begin{array}{c}\text { Falsos } \\
\text { negativos }\end{array}$ & VPP & VPN & $\mathrm{p}$ & $\begin{array}{l}\text { Certeza } \\
\text { diagnóstica }\end{array}$ & Карра \\
\hline Rupturas completas (\%) & 74 & 96 & 4 & 26 & 89 & 90 & $<0.001$ & 90 & 0.90 (muy buena) \\
\hline Rupturas parciales (\%) & 96 & 33 & 67 & 4 & 40 & 95 & 0.005 & 53 & 0.53 (moderada) \\
\hline
\end{tabular}


Llama la atención el alto porcentaje de pacientes a quienes se les etiquetó con un desgarro parcial del supraespinoso y los hallazgos quirúrgicos lo descartaron, lo que nos lleva a pensar que las consideraciones hechas por Naqvi y colaboradores y Zlatin MB y su equipo ${ }^{19,20,21,22}$ no son tomadas en cuenta al momento de llevar a cabo la elaboración del reporte de la IRM en los casos de desgarro del supraespinoso y por tanto, se sobrediagnostica esta patología.

Entre las fortalezas del estudio destaca que todos los procedimientos quirúrgicos fueron realizados por el mismo equipo quirúrgico, excluyendo cualquier diferencia con la técnica quirúrgica. El estudio analizó la concordancia de la imagen de resonancia magnética con la ruptura del tendón del músculo supraespinoso únicamente, lo cual lo hace más específico a esta patología.

Dentro de las limitaciones tenemos que es un estudio retrospectivo, sin cálculo de muestra, los estudios de resonancia magnética fueron realizados en diferentes centros de imagen, con diferentes radiólogos, por lo que el criterio podría haber variado al momento de interpretar las imágenes.

\section{Conclusiones}

La resonancia magnética en rupturas completas del tendón del músculo supraespinoso demuestra una buena sensibilidad y especificidad, un alto porcentaje de certeza diagnóstica, además de una muy buena concordancia con los hallazgos quirúrgicos. En las rupturas parciales no presenta la misma sensibilidad ni especificidad, además tiene una concordancia moderada, lo cual genera que estas lesiones estén sobrediagnosticadas.

Referencias

1. Dinnes J, Loveman E, McIntyre L, Waugh N. The effectiveness of diagnostic tests for the assessment of shoulder pain due to soft tissue disorders: a systematic review. NIHR Health Technology Assessment programme. Health Technol Assess. 2003; 7(29): 1-166.

2. Vitale MA, Vitale MG, Zivin JG, Braman JP, Bigliani LU, Flatow EL. Rotator cuff repair: an analysis of utility scores and cost-effectiveness. J Shoulder Elbow Surg. 2007; 16(2): 181-7.

3. Van Kampen DA, Van den Berg T, Van der Woude HJ, Castelein RM, Terwee CB, Willems WJ. The diagnostic value of the combination of patient characteristics, history and clinical shoulder test for the diagnosis of rotator cuff tear. J Orthop Surg Res. 2014; 9: 70.

4. Hapani H, Sood M, Trivedi A. Ultrasound, MRI and arthroscopic correlation of rotator cuff tears. International Journal of Contemporary Medical Reserch. 2017; 4(3): 77-83.
5. Bhatnagar A, Bhonsle S, Mehta S. Correlation between MRI and arthroscopy in diagnosis of shoulder pathology. J Clin Diagn Res. 2016; 10(2): RC18-21.

6. Youssef MA, Teima AH, Abduo YE, Salem LN. Ultrasonographic and MR diagnosis of rotator cuff disorders \& shoulder joint instability. Egypt J Radiol Nucl Med. 2013; 44(4): 835-44.

7. Abd-ElGawad EA, Ibraheem MA, Fouly EH. Evaluation of supraspinatus muscle tears by ultrasonography and magnetic resonance imaging in comparison with surgical findings. Egypt $J$ Radiol Nucl Med. 2013; 44(4): 829-34.

8. Abdulsahib HA, Abdelraoof MM. Role of MRI of diagnosis of rotator cuff tears. The Egyptian Journal of Hospital Medicine. 2018; 71(2): 2573-80.

9. Davidson JF, Burkhart SS, Richards DP, Campbell SE. Use of preoperative magnetic resonance imaging to predict rotator cuff tear pattern and method of repair. Arthroscopy. 2005; 21(12): 1428.

10. Ellman H, Kay SP, Wirth M. Arthroscopic treatment of full-thickness rotator cuff tears: 2- to 7-year follow-up study. Arthroscopy. 1993; 9(2): 195-200.

11. Collin P, Yoshida M, Delarue A, Lucas C, Jossaume T, Ladermann A. Evaluating postoperative rotator cuff healing: prospective comparison of MRI and ultrasound. Orthop Traumatol Surg Res. 2015; 101(6 Suppl): S265-8.

12. Yamamoto A, Takagishi K, Osawa T, Yanagawa T, Nakajima D, Shitara H, et al. Prevalence and risk factors of a rotator cuff tear in the general population. J Shoulder Elbow Surg. 2010; 19(1): 116-20.

13. Eljabu W, Klinger HM, Von Knoch M. The natural history of rotator cuff tears: a systematic review. Arch Orthop Trauma Surg. 2015; 135(8): 1055-61.

14. De Jesus JO, Parker L, Frangos AJ, Nazarian LN. Accuracy of MRI, MR arthrography, and ultrasound in the diagnosis of rotator cuff tears: a meta-analysis. AJR Am J Roentgenol. 2009; 192(6): 1701-7.

15. Tuite MJ, Yandow DR, DeSmet AA, Orwin JF, Quintana FA. Diagnosis of partial and complete rotator cuff tears using combined gradient echo and spin-echo imaging. Skeletal Radiol. 1994; 23(7): 541-5.

16. Rafii M, Firooznia H, Sherman O, Minkoff J, Weinreb J, Golimbu C, et al. Rotator cuff lesions: signal patterns at MR imaging. Radiology. 1990; 177(3): 817-23.

17. Reinus W, Shady K, MirowitzS TW. MR diagnosis of rotator cuff tears of the shoulder: value using T2-weighted fat-saturated images. AJR Am J Roentgenol. 1995; 164(6): 1451-5.

18. Sharma G, Bhandary S, Khandige G. MR Imaging of rotator cuff tears: correlation with arthroscopy. J Clin Diagn Res. 2017; 11(5): TC24-7.

19. Naqvi GA, Jadaan M, Harrington P. Accuracy of ultrasonography and magnetic resonance imaging for detection of full thickness rotator cuff tears. Int J Shoulder Surg. 2009; 3(4): 94-7.

20. Zlatin MB, Dlinka MK, Kressel HY. Magnetic resonance imaging of the shoulder. Magn Reson Q. 1989; 5(1): 3-22.

21. Zlatin MB, Dalinda MK. The glenohumeral joint. Top Magn Reson Imaging. 1989; 1(3): 1-13.

22. Gumina S. Rotator cuff tear: pathogenesis, evaluation and treatment. Cham: Springer International Publishing. 2017, 169-83. 